\title{
EZH2 and ALDH-1 mark breast epithelium at risk for breast cancer development
}

\author{
Lakshmi P Kunju ${ }^{1,5}$, Cynthia Cookingham ${ }^{1,5}$, Kathy A Toy ${ }^{2}$, Wei Chen ${ }^{3}$, Michael S Sabel ${ }^{2,4}$ \\ and Celina G Kleer ${ }^{1,2}$ \\ ${ }^{1}$ Department of Pathology, University of Michigan School of Medicine, Ann Arbor, MI, USA; \\ ${ }^{2}$ The Comprehensive Cancer Center, University of Michigan School of Medicine, Ann Arbor, MI, USA; \\ ${ }^{3}$ Department of Biostatistics, University of Michigan School of Medicine, Ann Arbor, MI, USA and \\ ${ }^{4}$ Department of Surgery, University of Michigan School of Medicine, Ann Arbor, MI, USA
}

\begin{abstract}
It is well established that benign proliferative lesions and atypical hyperplasia increase the risk of breast cancer, which can develop in either breast. At present, there is no radiological, pathological, or molecular marker capable of distinguishing which proliferative or atypical lesions will progress to carcinoma. EZH2, a protein involved in stem cell renewal and carcinogenesis is upregulated in the morphologically normal breast epithelium from BRCA1 mutation carriers. Here, we tested the hypothesis that EZH2 expression alone or in combination with the breast stem cell marker aldehyde dehydrogenase-1 (ALDH-1) may identify benign breast biopsies that progress to breast cancer in the future. Benign breast biopsy samples obtained from 59 women who subsequently developed (study group, $n=29$ ) or who did not develop (control group, $n=30$ ) breast cancer in the same time period were subjected to immunohistochemical analyses of EZH2 and ALDH-1 proteins. When present, EZH2 was expressed in the nuclei of benign epithelial cells, whereas ALDH-1 was expressed in the cytoplasm of epithelial cells and/or in the stroma. EZH2, epithelial ALDH-1, and expanded stromal ALDH-1-positive cells were present in 95, 43, and 69\%, respectively, of study group biopsies, compared with 16,13 , and $37 \%$, respectively, of control biopsies $(P<0.05$ for all). The mean percentage of EZH2-positive cells was higher in the study group than in the control group (34 and $6 \%$, respectively). EZH2 expression was associated with breast cancer development $\left(P=8.2 \times 10^{-6}\right)$ and with younger age at cancer diagnosis $(P=0.0086)$. Both stromal and epithelial ALDH-1 were associated with development of breast cancer $(P=0.001$ and $P=0.049$, respectively). Our study provides first evidence that EZH2 and epithelial and stromal ALDH-1 detection in benign breast biopsies may predict increased risk for breast cancer, with implications for breast cancer prevention.
\end{abstract}

Modern Pathology (2011) 24, 786-793; doi:10.1038/modpathol.2011.8; published online 11 March 2011

Keywords: breast cancer risk; EZH2; predictive biomarker; prevention; stem cells; ALDH-1

Benign breast disease is an important risk factor for later breast cancer, which can develop in either breast. $^{1,2}$ Compelling data indicate that atypical ductal hyperplasia, atypical lobular hyperplasia, and proliferative lesions (prominent ductal hyperplasia, papilloma, radial scar, and sclerosing ade-

Correspondence: Dr CG Kleer, MD, Department of Pathology, University of Michigan School of Medicine, 4217 Comprehensive Cancer Center, 1500 East Medical Center Drive, Ann Arbor, MI 48109, USA.

E-mail: kleer@umich.edu

This study was presented in part at the 98th United States and Canadian Academy of Pathology (USCAP) Meeting, Boston, MA, February 2009.

${ }^{5}$ These authors contributed equally to this work and are considered co-first authors.

Received 30 June 2010; revised 9 November 2010; accepted 21 November 2010; published online 11 March 2011 nosis) increase the risk of breast cancer. ${ }^{2,3}$ The identification of benign breast disease has become more common as the use of mammography has increased. Having accurate risk estimates for women who receive this diagnosis is necessary but not available at present.

The Polycomb group (PcG) proteins form chromatin-modifying complexes essential for embryonic development and stem cell renewal and are deregulated in cancer. ${ }^{4}$ Increasing evidence suggests that the oncogenic role of the PCG protein EZH2 may be ascribed to its role in stem cell maintenance. ${ }^{5,6}$ Our group and other investigators have found that EZH2 has potent oncogenic properties in the breast. ${ }^{5,7-12}$ We have reported that EZH2 protein expression is elevated in the benign-appearing breast epithelium from prophylactic mastectomies of BRCA1 mutation carriers. ${ }^{13}$ Holst et $a l^{14}$ showed that a small number 
of cells from the normal breast epithelium can bypass senescence in vitro, and are associated with pre-neoplastic phenotypes. These cells exist in vivo as well, as rare foci of morphologically normal cells in disease-free breast. The same group recently showed that loss of p16INK4A results in overexpression of EZH2, recruitment of DNA methyltransferases, and DNA hypermethylation, and proposed that these epigenetic events are early events in breast cancer initiation. ${ }^{15}$

Activation of the enzymatic activity of aldehyde dehydrogenase-1 (ALDH-1) in the breast epithelium has been shown to be a marker of breast cancer stem cells and to have a functional role in stem cell renewal and differentiation. ${ }^{16-18}$ ALDH-1 protein expression in breast carcinomas detected by immunohistochemistry was associated with poor clinical outcome. ${ }^{6,16,17}$ We have noted that ALDH-1 is expressed by intralobular stromal cells, and that its expression is expanded beyond the intralobular stroma in tissue samples. In this study, we tested the hypothesis that the expression of EZH2 alone or in combination with epithelial and/or stromal ALDH-1 protein may identify benign proliferative and atypical lesions at increased risk for breast cancer.

\section{Materials and methods}

\section{Case Selection}

The Surgical Pathology files at the University of Michigan were searched after obtaining Institutional Board Review approval for breast tissue samples from women who had undergone a breast biopsy for a benign breast lesion between 1970 and 2003 and who subsequently developed breast cancer (study group). As a control group, we included benign breast samples obtained from women who had undergone a breast biopsy during the same time frame and did not subsequently develop breast cancer.

\section{Morphological Review}

Histopathological evaluation was performed independently and blindly by three practicing pathologists (LPK, CC, and CGK) with expertise in breast disease. Differences in opinion in rare instances were resolved by consensus evaluation of the case with the senior pathologist (CGK). Morphological parameters evaluated in this study included: (1) the presence of non-proliferative fibrocystic changes, including simple cysts, apocrine metaplasia, and stromal fibrosis; (2) proliferative lesions, including adenosis, papillomas, and intraductal hyperplasia; and (3) the presence of epithelial atypia, including flat epithelial atypia and atypical ductal hyperplasia. In the current study, atypical ductal hyperplasia and flat epithelial atypia were diagnosed following the criteria established by the World Health
Organization $^{19}$ and according to the classification system outlined by Schnitt and Vincent-Salomon, ${ }^{20}$ respectively.

At present, there is no agreement on whether quantitative criteria should be applied to distinguish atypical ductal hyperplasia from low-grade ductal carcinoma in situ. Thus, atypical ductal hyperplasia was diagnosed when there was a proliferation of evenly distributed monomorphic cells growing in different patterns (micropapillary, cribriform), but that coexisted with usual ductal hyperplasia, and/or there was partial ductal involvement. Flat epithelial atypia lesions were characterized by variably distended terminal duct lobular units in which native epithelial cells are replaced by one to several layers of a monotonous, atypical cuboidal to columnar cell population with apical snouts, secretory or floccular material in the lumen, often with microcalcifications. Flat epithelial atypia by definition, unlike atypical ductal hyperplasia, has a flat growth pattern with no complex architecture.

\section{Clinical Information}

Relevant clinical information including age of patients at the time of breast biopsy with benign breast disease, as well as age at time of diagnosis of cancer and details of breast cancer such as type, size, Nottingham grade, TNM stage, hormone receptors (estrogen receptor (ER) and progesterone receptor) status, HER-2/neu status, and axillary lymph-node involvement were recorded for all cases.

\section{Immunohistochemistry}

Immunohistochemistry was performed on tissue sections with the standard biotin-avidin complex technique using a monoclonal antibody against EZH2 (1:300, BD Biosciences, San Diego, CA) and ALDH-1 (1: 5000, BD Biosciences). EZH2 expression was recorded as the percentage of epithelial cells with nuclear expression as performed previously. ${ }^{13}$ The epithelial expression of ALDH-1 was scored as either positive or negative. ALDH-1 staining when present in epithelial cells was clearly cytoplasmic and noted in at least $5 \%$ of epithelial cells in both groups. ${ }^{16}$ Stromal ALDH-1 expression was considered expanded when it extended beyond the intralobular stroma into the interlobular stroma. EZH2 and ALDH-1 expression was evaluated in epithelial cells, including proliferative lesions and atypical hyperplasia. Foci with maximum intensity staining were scored. Slides were reviewed in a blinded manner.

\section{Statistical Analysis}

ALDH-1 scores of the study and control groups were tabulated, and Fisher's exact test was performed to 
assess the association between ALDH-1 and cancer development. In addition to this, treating ALDH-1 expression as ordinary, logistic regression analysis was performed. Mean EZH2 expression was compared between the study and control groups using an unpaired two-sample $t$-test. The possible association between EZH2 expression and group status was analyzed using logistic regression. On the basis of this logistic prediction model, a receiver operating characteristic (ROC) curve was plotted to evaluate the performance of the model. A leave-one-out cross-validation procedure was applied to avoid overfitting. Additional clinical variables were compared between the two groups. Similarly, Fisher's exact test and unpaired two-sample $t$-test were applied to categorical and continuous variables, respectively.

\section{Results}

\section{Histopathological and Clinical Characteristics of the Study Cohorts}

To test the hypothesis that EZH2 expression alone or in combination with the breast cancer stem cell marker ALDH-1 may identify benign breast epithelium, proliferative and atypical lesions that may progress to breast cancer in the future, we studied benign breast biopsy samples obtained from 59 women who subsequently developed (study group, $n=29$ ) or who did not develop (control group, $n=30$ ) breast cancer in the same time period. These women did not have a personal history of breast cancer. Detailed histopathological and immunohistochemical analyses for EZH2 and ALDH-1 were performed. The histological classifications included no significant abnormality, non-proliferative fibrocystic changes, proliferative fibrocystic changes, and epithelial atypia (atypical ductal hyperplasia and/or flat epithelial atypia).

Benign biopsy samples from women in the study group had increased incidence of proliferative and atypical lesions compared with controls and exceeded those reported in the general population. ${ }^{19}$ The study group showed proliferative lesions and epithelial atypia in 9 of $29(31 \%)$ and in 7 of 29 $(24 \%)$ cases, respectively, compared with 6 of 30 $(24 \%)$ and 3 of $30(10 \%)$, respectively, in the control group. These differences were not statistically significant $\left(\chi^{2}\right.$ test, $P=0.31 ;$ Fisher's exact test, $P=0.24)$ (Table 1).

The mean age of women at the time of initial benign biopsy was higher in the study group than in the control group $(52.3 \pm 11.9$ years and $46.9 \pm 10.6$ years, respectively). The mean age at the time of cancer diagnosis of women in the study group was $59 \pm 11.1$ years, with a mean time frame of $6.7 \pm 4.5$ years to develop cancer from the initial benign biopsy. The mean follow-up time for control group was $8.3 \pm 5.3$ years. Clinical and histological features
Table 1 Clinical characteristics and histological findings at the time of initial biopsy in the study and control groups

\begin{tabular}{lcc}
\hline & $\begin{array}{c}\text { Study group } \\
(\mathrm{n}=29)\end{array}$ & $\begin{array}{c}\text { Control group } \\
(\mathrm{n}=30)\end{array}$ \\
\hline Age of benign (mean+s.d.) & $52.3 \pm 11.9$ & $46.9 \pm 10.6$ \\
Age of cancer & $59.0 \pm 11.1$ & $\mathrm{NA}$ \\
Years of development (mean+s.d.) & $6.7 \pm 4.5$ & $\mathrm{NA}$ \\
& & \\
Menopausal status & $4(14 \%)$ & $16(53 \%)$ \\
Pre-menopausal & $7(25 \%)$ & $5(17 \%)$ \\
Peri-menopausal & $18(62 \%)$ & $9(30 \%)$ \\
Post-menopausal & & \\
& $5(17 \%)$ & $13(43 \%)$ \\
Pathological findings on biopsy & $8(28 \%)$ & $11(37 \%)$ \\
$\quad$ No significant abnormality & $3(20 \%)$ \\
Non-proliferative fibrocystic & $2(7 \%)$ & $1(3 \%)$ \\
Proliferative fibrocystic & $4(14 \%)$ & $1(3 \%)$ \\
$\quad \begin{array}{l}\text { Atypical ductal hyperplasia } \\
\text { Atypical ductal hyperplasia and }\end{array}$ & $4(3 \%)$ & $1(3 \%)$ \\
flat epithelial atypia & $1(3 \%)$ & \\
$\quad$ Flat epithelial atypia &
\end{tabular}

NA, not applicable.

at the time of initial breast biopsy are shown in Table 1.

Most breast carcinomas that developed in the study group were invasive ductal $(86 \%)$, predominantly moderate to high grade (Nottingham grade 2 or 3, 66\%), and ER positive (69\%). Table 2 summarizes the clinical and pathological characteristics of the invasive carcinomas.

\section{The Percentage of EZH2-positive Cells Marks Increased Breast Cancer Risk and Younger Age at Breast Cancer Diagnosis}

EZH2 expression was observed mainly in the nucleus of epithelial cells as described previously. ${ }^{8,9}$ EZH2-positive cells were present in 95\% of biopsies in the study group compared with $16 \%$ of control biopsies. The mean percentage of EZH2-positive cells in the study group was $34 \%( \pm 27 \%)$ compared with $6 \%( \pm 10 \%)$ in the control group $\left(P=8.2 \times 10^{-6}\right.$ and $P=7.3 \times 10^{-4}$, two-sample $t$-test and logistic regression, respectively). EZH2 expression was associated with development of carcinoma in the absence of atypical ductal hyperplasia in the biopsy (logistic regression, $P=0.004$ ). Figure 1a shows representative pictures of benign breast biopsies expressing EZH2. A logistic prediction model based on EZH2 expression further confirmed our observation that EZH2 expression may predict future breast cancer diagnosis; when plotted on an ROC curve, the area under the curve for EZH2 expression was 0.88 (Figure $1 b$ ).

We next evaluated the relationship between EZH2 expression and patient age at the time of benign breast biopsy and cancer diagnosis, and also the time interval required to develop breast cancer after a benign biopsy. EZH2 upregulation was significantly associated with younger age at the time of benign breast biopsy $(P=0.002)$ and younger age at 
Table 2 Pathological features of breast carcinomas that developed in the 29 women in study group

\begin{tabular}{|c|c|}
\hline \multicolumn{2}{|l|}{ Size of tumors $(\mathrm{cm})$} \\
\hline Median & 1.2 \\
\hline Mean & 1.4 \\
\hline Range & $0.5-2.5$ \\
\hline \multicolumn{2}{|l|}{ Type of tumor $(n=29)$} \\
\hline Ductal & $25(86 \%)$ \\
\hline Lobular & $3(10 \%)$ \\
\hline Mixed ductal/lobular & $1(4 \%)$ \\
\hline \multicolumn{2}{|l|}{ Nottingham grade } \\
\hline Grade 1 & $10(34 \%)$ \\
\hline Grade 2 & $13(45 \%)$ \\
\hline Grade 3 & $6(21 \%)$ \\
\hline \multicolumn{2}{|l|}{ Hormone receptors } \\
\hline \multicolumn{2}{|l|}{$E R$} \\
\hline Negative & $9(31 \%)$ \\
\hline Positive & $20(69 \%)$ \\
\hline \multicolumn{2}{|l|}{$P R$} \\
\hline Negative & $12(42 \%)$ \\
\hline Positive & $17(58 \%)$ \\
\hline \multicolumn{2}{|l|}{ HER-2/Neu } \\
\hline Not overexpressed & $28(96 \%)$ \\
\hline Overexpressed & $1(4 \%)$ \\
\hline \multicolumn{2}{|l|}{ TNM stage } \\
\hline $\mathrm{I}$ & $20(64 \%)$ \\
\hline II & $5(17 \%)$ \\
\hline III & $3(10 \%)$ \\
\hline IV & $1(4 \%)$ \\
\hline \multicolumn{2}{|l|}{ Lymph node status } \\
\hline Positive & $5(17 \%)$ \\
\hline Negative & $21(72 \%)$ \\
\hline Not assessed & $3(10 \%)$ \\
\hline
\end{tabular}

the time of cancer diagnosis $(P=0.0086)$ in the study group. The mean interval to develop breast cancer after a benign biopsy was 6.7 years. EZH2 expression was not associated with patient age at the time of initial benign breast biopsy in the control group.

\section{ALDH-1 Expression is Associated with Increased Breast Cancer Risk}

ALDH-1 showed cytoplasmic expression in epithelial cells as described previously. ${ }^{6,16,17}$ We noted that stromal cells also express ALDH-1. Figure 2 shows examples of ALDH-1 expression in benign biopsies. We evaluated both epithelial and stromal ALDH-1 expression in benign biopsy samples obtained from the study and control groups. Epithelial ALDH-1 was present in 43 and $13 \%$ of biopsies in the study and control groups, respectively. There was a modest association between ALDH-1 and cancer development (Fisher's exact test, $P=0.049$; logistic regression, $P=0.02$ ). Although stromal ALDH-1 staining was seen in intralobular stromal cells in most cases, the expansion of this population was significantly associated with increased risk for breast cancer (Fischer's exact test, $P=0.0099$ ) (Figure 2a-d). It must be noted that both epithelial and stromal ALDH-1 were associated with development of carcinoma in the absence of atypical ductal hyperplasia in the biopsy (logistic regression, $P=0.02$ and $P=0.007$, respectively). When analyzed together, epithelial and/or stromal ALDH-1 did not add significant value to the already significant effect of EZH2 in predicting breast cancer development. These findings are summarized in Table 3.

\section{Discussion}

In the current era of mammographic screening, an increasing number of women are being diagnosed with benign breast lesions. Although the presence of atypical hyperplasia and proliferative lesions without atypia, age at the time of biopsy, and/or family history are determinants of breast cancer risk in women with benign breast biopsies, there are no means to identify which patients will progress to carcinoma. ${ }^{1-3}$ Identification of clinically useful molecular markers of increased breast cancer risk, especially those that can be applied in precancerous states would help guide clinical decisions.

The PcG protein EZH2 functions in multimeric complexes that regulate gene expression to maintain cell identity and differentiation. EZH2 has been implicated in stem cell regulation and malignancy, including breast cancer. ${ }^{7,9-11,21-24} \mathrm{~A}$ recent report has provided evidence that EZH2 is important for tissuederived stem cell maintenance. ${ }^{10}$ Our group has discovered that EZH2 is upregulated in morphologically normal breast epithelium at increased risk for breast cancer. ${ }^{13,25}$ In particular, we have shown that EZH2 is overexpressed in prophylactic mastectomies from BRCA1 carriers compared with normal breasts from women who underwent a breast reduction surgery and had no personal history of breast cancer. ${ }^{13,25}$ These findings suggest that EZH2 upregulation may occur in histologically normal epithelium at higher risk of breast cancer and prompted us to undertake this study.

Several insights emerge from our study. Benign breast biopsy samples obtained from women who subsequently develop breast cancer exhibited proliferative breast disease, atypical ductal hyperplasia, and/or flat epithelial atypia more frequently than did biopsy samples from women who did not develop breast cancer. This finding fully supports previous studies, which have shown that atypical hyperplasia confers the highest relative risk of breast cancer in women with benign breast disease. ${ }^{2,3}$

An important novel observation is that EZH2 is overexpressed in benign breast biopsies of women who subsequently develop breast cancer compared with the control group (95 vs $16 \%, P=8.2 \times 10^{-6}$ ). Although the ability of EZH2 expression to predict 
a

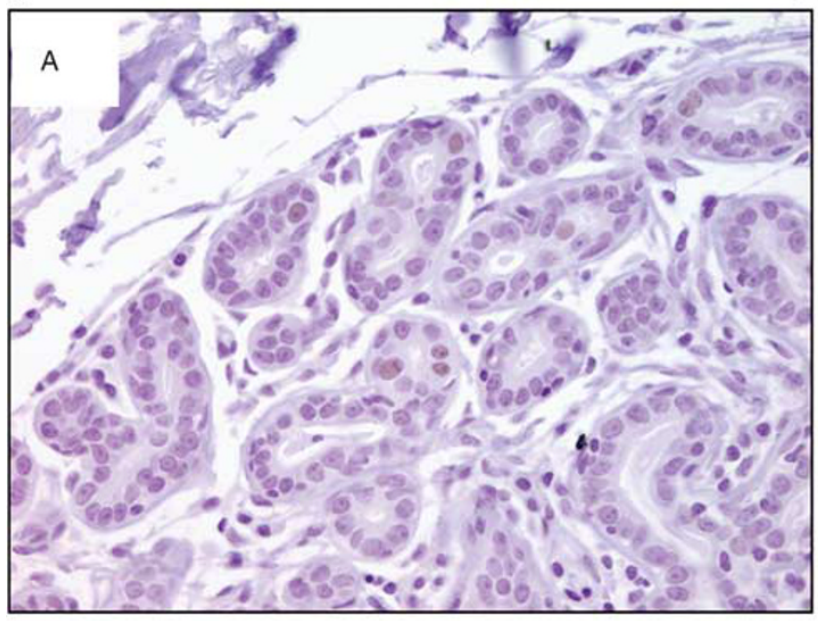

EZH2

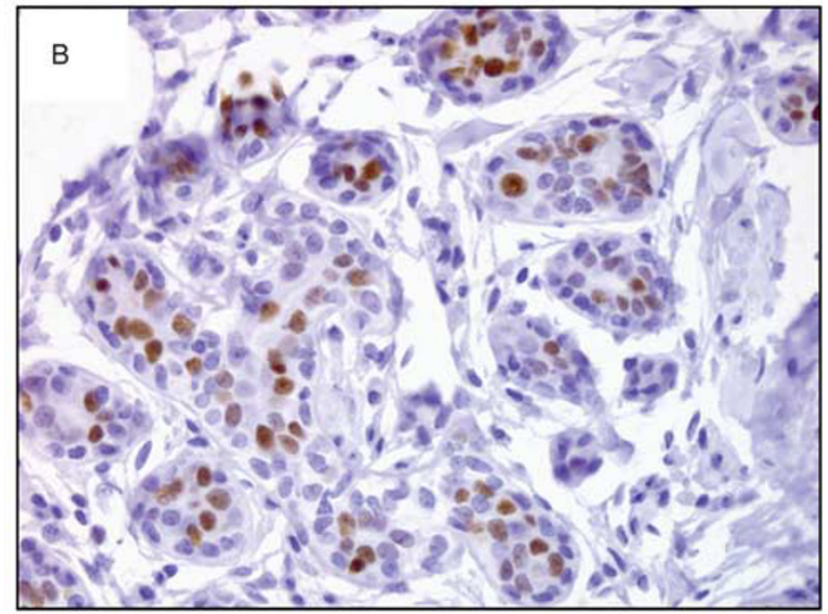

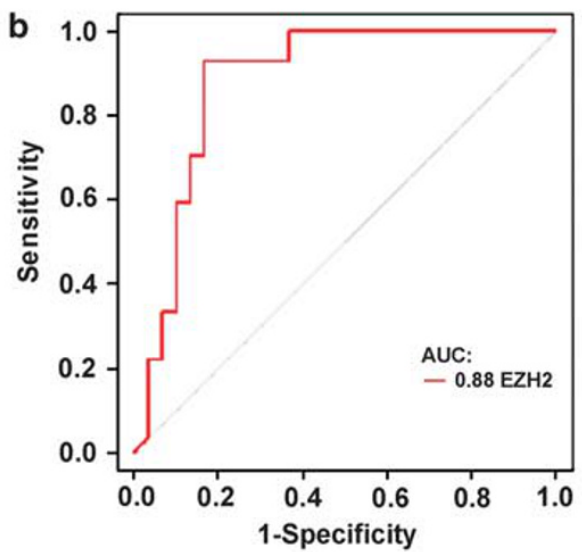

Figure 1 EZH2 expression in benign breast and breast cancer development. (a) Representative pictures of benign breast lobules analyzed for EZH2 protein expression; (A) benign lobule with rare cells expressing nuclear EZH2 and (B) increased EZH2 nuclear expression in epithelial cells. (b) Receiver operating curve showing that EZH2 detection has high sensitivity and specificity in predicting which women with benign breast biopsies develop carcinoma in the future.

future breast cancer development may be linked to cell proliferation, its role as a predictor of breast cancer may depend on its effect on stem cell survival and alteration of DNA repair pathways. ${ }^{10,11,22,26,27}$

Young age at breast cancer diagnosis has been associated with worse outcome in breast cancer. Younger women exhibit worse survival than do older women. ${ }^{28,29}$ This association is likely to be related to tumor biology as breast carcinomas among young women are more likely lymph node positive, hormone receptor negative, and possess complex molecular alterations. ${ }^{30,31}$ We have previously reported that EZH2 overexpression is associated with worse prognosis in patients with breast cancer. ${ }^{8}$ Here, we found that the EZH2 protein is significantly overexpressed in younger women both at the time of benign $(P=0.0023)$ and breast cancer diagnoses $(P=0.0086)$. Taken together, our data allow us to postulate that EZH2 may have distinct roles at different stages of breast cancer progression, in the benign epithelium and in breast cancer cells. We are currently investigating this hypothesis.
In view of the potential role of EZH2 in cancer stem cells, ${ }^{10}$ we evaluated the utility of the breast cancer stem cell marker candidate ALDH-1 in identifying benign breast tissue at increased risk for carcinoma. Measurement of ALDH-1 enzymatic activity has been shown to distinguish benign and breast cancer stem cells and other neoplasms, including human hematopoietic malignancies. ${ }^{32,33}$ ALDH-1 has a functional role in stem cell differentiation. It has been demonstrated that expression of ALDH-1 in the cytoplasm of cancer cells is associated with poor clinical outcome in patients with breast cancer and that high ALDH-1 activity selects for both normal and tumorigenic human mammary epithelial cells with stem/progenitor properties. ${ }^{16}$ Recently, Resetkova et al $l^{6}$ showed that tumoral stromal expression of ALDH-1 by immunohistochemistry was associated with survival of triple-negative invasive breast carcinomas. Here, we report that ALDH-1 is expressed in epithelial and stromal cells in benign breast tissues, and discovered that ALDH-1 positivity in breast epithelial cells is associated with increased risk of breast 
ALDH-1
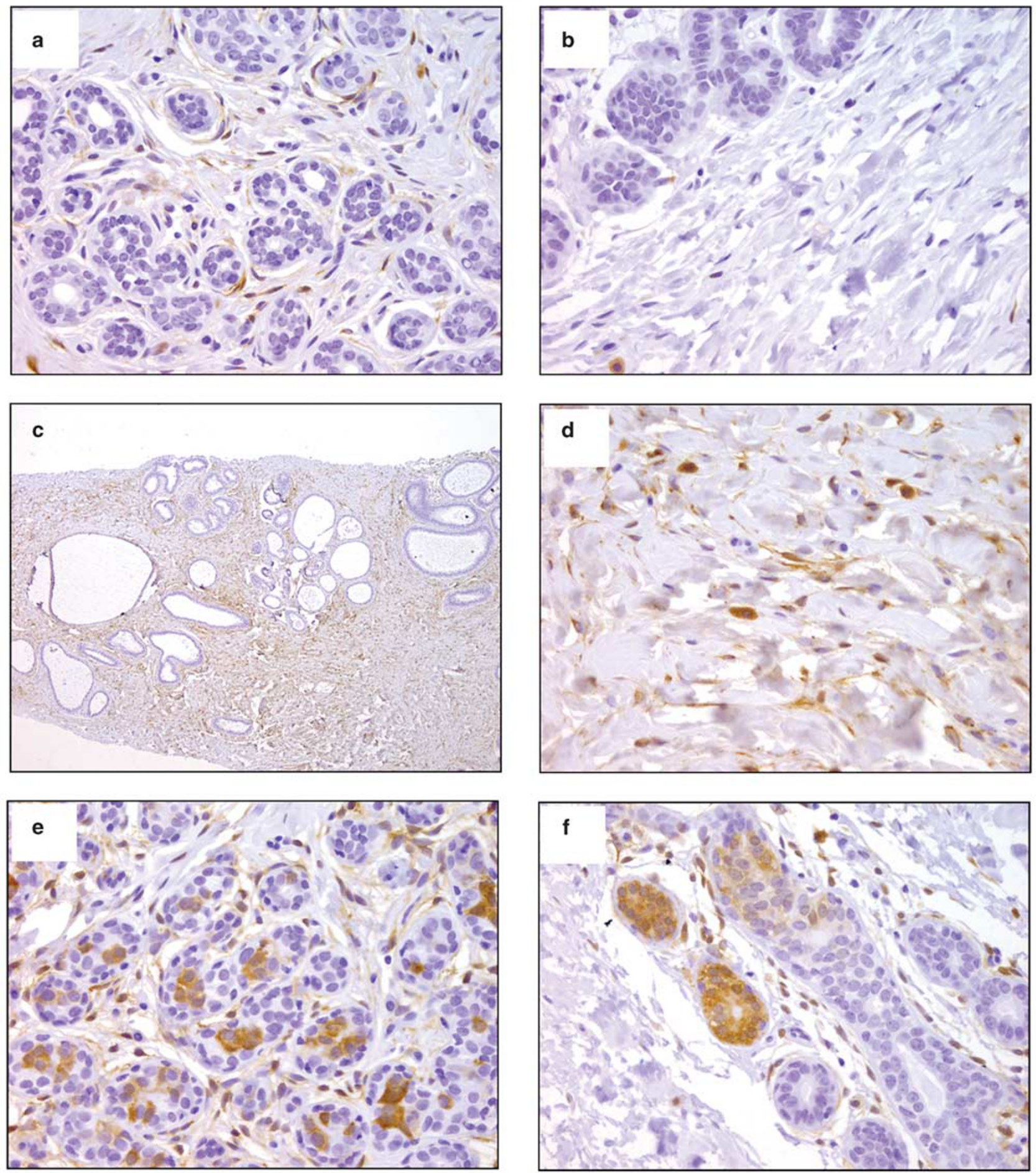

Figure 2 ALDH-1 expression in epithelial and stromal cells of benign breast biopsies. Representative pictures showing: (a and b) Control group biopsy showing that ALDH-1 is normally expressed in stromal cells composing the intralobular stroma, and is absent in the epithelium. It must be noted that ALDH-1 is rarely expressed in interlobular stromal cells (panel b). (c and d). Study group biopsy showing expansion of ALDH-1 stromal expression. ALDH-1 is expressed by interlobular stromal cells in this biopsy. (e and f) Two study group biopsies illustrating ALDH-1 expression in the cytoplasm of epithelial cells. We found that ALDH-1 expression in the epithelium is focal with in the lobule.

cancer. It must be noted that expansion of the stromal cell population expressing ALDH-1 beyond the intralobular stroma is also associated with cancer development. Taken together, these data suggest a role for the microenvironment in promoting tumor development before morphological 
Table 3 Expression of EZH2, epithelial and stromal ALDH-1 in benign biopsies

\begin{tabular}{lccc}
\hline & $\begin{array}{c}\text { Study } \\
\text { group } \\
(\mathrm{n}=29)\end{array}$ & $\begin{array}{c}\text { Control } \\
\text { group } \\
(\mathrm{n}=30)\end{array}$ & P-value \\
& & & \\
\hline EZH2 & $95 \%$ & $16 \%$ & \\
EZH2+biopsies (\%) & $33.6+27.2$ & $5.6+9.9$ & $8.2 \times 10^{6 \mathrm{a}}$ \\
EZH2+cells (mean+s.d.) & & & \\
$\quad$ & & & \\
ALDH-1 (number, \%) & $15(52 \%)$ & $23(77 \%)$ & \\
$\quad$ Epithelial & & \\
$\quad$ Negative & $14(48 \%)$ & $7(23 \%)$ & $0.049^{\mathrm{b}}$ \\
$\quad$ Positive & $10(31 \%)$ & $19(63 \%)$ & \\
$\quad$ Stromal & Intralobular & & \\
$\quad$ Expanded & $20(69 \%)$ & $11(37 \%)$ & $0.0099^{\mathrm{b}}$ \\
\hline
\end{tabular}

${ }^{\mathrm{a}}$ Values of the two-sample test are represented here.

${ }^{\mathrm{b}}$ Values of Fisher's exact test are represented here.

abnormalities of carcinoma become evident. These data also support the utility of immunohistochemistry in the evaluation of ALDH-1 as a putative marker of breast cancer stem cells.

The limited number of cases in our study precludes multivariable statistical analyses to investigate whether EZH2 and/or ALDH-1 are associated with breast cancer independently of epithelial atypias and other clinical features. However, when biopsies containing atypical ductal hyperplasia were excluded from the analysis, EZH2, epithelial and stromal ALDH-1 remained significantly associated with breast cancer diagnosis.

In summary, benign breast biopsies in women who subsequently develop breast cancer show increased incidence of proliferative and atypical lesions compared with controls. Epithelial atypia including atypical ductal hyperplasia and/or flat epithelial atypia are more common in these biopsies and exceed those reported in the general population. We have characterized for the first time the expression of EZH2 and candidate breast stem cell marker ALDH-1 in benign breast biopsies. Our data show that EZH2 and ALDH-1 upregulation is frequently noted in high-risk proliferative lesions in benign breast and is significantly associated with subsequent development of breast cancer at a younger age. Our data pave the way for larger prospective validation studies, as these findings may have clinical implications in the evaluation and management of women with benign breast biopsies.

\section{Acknowledgements}

This work was supported by NIH grants 2R01CA107469, R01CA125577, and U01CA154224 (to CGK), and by the National Institutes of Health through the University of Michigan's Cancer Center Support Grant (5 P30 CA46592).

\section{Disclosure/conflict of interest}

The authors declare no conflict of interest.

\section{References}

1 Dupont WD, Page DL. Risk factors for breast cancer in women with proliferative breast disease. N Engl J Med 1985;17:312: 146-51.

2 Hartmann LC, Sellers TA, Frost $\mathrm{MH}$, et al. Benign breast disease and the risk of breast cancer. N Engl J Med 2005;353:229-237.

3 Degnim AC, Visscher DW, Berman HK, et al. Stratification of breast cancer risk in women with atypia: a Mayo cohort study. J Clin Oncol 2007;25:2671-2677.

4 Jacobs JJ, Kieboom K, Marino S, et al. The oncogene and Polycomb-group gene bmi-1 regulates cell proliferation and senescence through the ink4a locus. Nature 1999;397:164-168.

5 Pietersen AM, Horlings HM, Hauptmann M, et al. EZH2 and BMI1 inversely correlate with prognosis and TP53 mutation in breast cancer. Breast Cancer Res 2008;10:R109.

6 Resetkova E, Reis-Filho JS, Jain RK, et al. Prognostic impact of ALDH1 in breast cancer: a story of stem cells and tumor microenvironment. Breast Cancer Res Treat 2010;123:97-108.

7 Bachmann IM, Halvorsen OJ, Collett K, et al. EZH2 expression is associated with high proliferation rate and aggressive tumor subgroups in cutaneous melanoma and cancers of the endometrium, prostate, and breast. J Clin Oncol 2006;24:268-273.

8 Kleer CG, Cao Q, Varambally S, et al. EZH2 is a marker of aggressive breast cancer and promotes neoplastic transformation of breast epithelial cells. Proc Natl Acad Sci USA 2003;100:11606-11611.

9 Raaphorst FM, Meijer CJ, Fieret E, et al. Poorly differentiated breast carcinoma is associated with increased expression of the human polycomb group EZH2 gene. Neoplasia 2003;5:481-488.

10 Ezhkova E, Pasolli HA, Parker JS, et al. Ezh2 orchestrates gene expression for the stepwise differentiation of tissue-specific stem cells. Cell 2009;136: 1122-1135.

11 Gonzalez ME, Li X, Toy K, et al. Downregulation of EZH2 decreases growth of estrogen receptor-negative invasive breast carcinoma and requires BRCA1. Oncogene 2009;28:843-853.

12 Puppe J, Drost R, Liu X, et al. BRCA1-deficient mammary tumor cells are dependent on EZH2 expression and sensitive to Polycomb repressive complex 2inhibitor 3-deazaneplanocin A. Breast Cancer Res 2009;11:R63.

13 Ding L, Erdmann C, Chinnaiyan AM, et al. Identification of EZH2 as a molecular marker for a precancerous state in morphologically normal breast tissues. Cancer Res 2006;66:4095-4099.

14 Holst CR, Nuovo GJ, Esteller M, et al. Methylation of p16(INK4a) promoters occurs in vivo in histologically normal human mammary epithelia. Cancer Res 2003;63:1596-1601.

15 Reynolds PA, Sigaroudinia M, Zardo G, et al. Tumor suppressor p16INK4A regulates polycomb-mediated DNA hypermethylation in human mammary epithelial cells. J Biol Chem 2006;281:24790-24802. 
16 Ginestier C, Hur MH, Charafe-Jauffret E, et al. ALDH1 is a marker of normal and malignant human mammary stem cells and a predictor of poor clinical outcome. Cell Stem Cell 2007;1:555-567.

17 Neumeister V, Agarwal S, Bordeaux J, et al. In situ identification of putative cancer stem cells by multiplexing ALDH1, CD44, and cytokeratin identifies breast cancer patients with poor prognosis. Am J Pathol 2010;176:2131-2138.

18 Dontu G, Abdallah WM, Foley JM, et al. In vitro propagation and transcriptional profiling of human mammary stem/progenitor cells. Genes Dev 2003;17:1253-1270.

19 Tavassoli FA, Devilee P. Pathology and Genetics of Tumors of the Breast and Female Genital Organs. IARC Press: Lyon, France, 2003.

20 Schnitt SJ, Vincent-Salomon A. Columnar cell lesions of the breast. Adv Anat Pathol 2003;10:113-124.

21 Laible G, Wolf A, Dorn R, et al. Mammalian homologues of the Polycomb-group gene enhancer of zeste mediate gene silencing in Drosophila heterochromatin and at S. cerevisiae telomeres. EMBO J 1997;16: 3219-3232.

22 Zeidler M, Varambally S, Cao Q, et al. The Polycomb group protein EZH2 impairs DNA repair in breast epithelial cells. Neoplasia 2005;7:1011-1019.

23 Bracken AP, Dietrich N, Pasini D, et al. Genomewide mapping of Polycomb target genes unravels their roles in cell fate transitions. Genes Dev 2006;20: 1123-1136.

24 Collett K, Eide GE, Arnes J, et al. Expression of enhancer of zeste homologue 2 is significantly associated with increased tumor cell proliferation and is a marker of aggressive breast cancer. Clin Cancer Res 2006;12:1168-1174.
25 Ding L, Kleer CG. Enhancer of Zeste 2 as a marker of preneoplastic progression in the breast. Cancer Res 2006;66:9352-9355.

26 Aoto T, Saitoh N, Sakamoto Y, et al. Polycomb group protein-associated chromatin is reproduced in postmitotic G1 phase and required for S-phase progression. J Biol Chem 2008;283:18905-18915.

27 Cao Q, Yu J, Dhanasekaran SM, et al. Repression of E-cadherin by the polycomb group protein EZH2 in cancer. Oncogene 2008;27:7274-7284.

28 Institut Gustave-Roussy Breast Cancer Group. Arriagada R, Le MG, Rochard F, et al. Conservative treatment versus mastectomy in early breast cancer: patterns of failure with 15 years of follow-up data. J Clin Oncol 1996;14:1558-1564.

29 Fisher B, Anderson S, Tan-Chiu E, et al. Tamoxifen and chemotherapy for axillary node-negative, estrogen receptor-negative breast cancer: findings from National Surgical Adjuvant Breast and Bowel Project B-23. J Clin Oncol 2001;19:931-942.

30 Kroman N, Jensen MB, Wohlfahrt J, et al. Factors influencing the effect of age on prognosis in breast cancer: population based study. BMJ 2000;320: 474-478.

31 Kollias J, Elston CW, Ellis IO, et al. Early-onset breast cancer-histopathological and prognostic considerations. Br J Cancer 1997;75:1318-1323.

32 Hess DA, Wirthlin L, Craft TP, et al. Selection based on CD133 and high aldehyde dehydrogenase activity isolates long-term reconstituting human hematopoietic stem cells. Blood 2006;107:2162-2169.

33 Storms RW, Trujillo AP, Springer JB, et al. Isolation of primitive human hematopoietic progenitors on the basis of aldehyde dehydrogenase activity. Proc Natl Acad Sci USA 1999;96:9118-9123. 